\title{
Genetic evaluation of in-line recorded milkability from milking parlors and automatic milking systems
}

\author{
C. Carlström, ${ }^{* 1}$ E. Strandberg, ${ }^{*}$ K. Johansson,† G. Pettersson, † H. Stålhammar,§ and J. Philipsson* \\ *Department of Animal Breeding and Genetics, Swedish University of Agricultural Sciences, PO Box 7023, SE-750 07 Uppsala, Sweden \\ †Växa Sweden, Box 7023, S-75007 Uppsala, Sweden \\ fDepartment of Animal Nutrition and Management, Swedish University of Agricultural Sciences, SE-753 23 Uppsala, Sweden \\ §Viking Genetics, Box 64, S-532 21 Skara, Sweden
}

\section{ABSTRACT}

The overall objective of this study was to assess the use of in-line recorded milkability information from dairy herds with conventional milking parlors (CMP) and from herds with automatic milking systems (AMS) for genetic evaluation. Some genetic parameters were previously studied on AMS data for 2,053 Swedish Holstein (SH) and 1,749 Swedish Red (SR) cows in 19 herds. These data were combined in the present paper with milkability information from 74 herds with CMP, including 11,123 SH cows and 7,554 SR cows. Genetic parameters were estimated for the CMP data and genetic correlations were estimated between milkability traits measured in the 2 systems. Average flow rate and milking time were derived and used as similar milkability traits for both systems, whereas box time was used only for AMS herds. Estimated heritabilities were in the range from 0.24 to 0.49 . Even though the traits were differently defined in the 2 milking systems, the corresponding traits recorded in AMS and CMP were genetically closely related (0.93-1.00). Similarly, close genetic relationships were shown between milkability traits in different lactations in both breeds (0.93-0.99). Thus, it should be possible to treat milkability traits in different lactations and from different milking systems as the same traits in genetic evaluations. The various milkability traits were also highly genetically correlated, indicating that the inclusion of just one trait in the genetic selection program would efficiently select for milkability without the need to consider all measures. Comparisons of repeatability and random regression models, combining all information from the 2 systems for genetic evaluation, were done to find the most suitable model for genetic evaluation purposes. Even though the random regression models were favored in the formal model tests to evaluate suitability, correlation coefficients between test-days within lactation were

Received April 21, 2013.

Accepted October 7, 2013.

${ }^{1}$ Corresponding author: caroline.carlstrom@slu.se high (0.7-0.8) and small differences in breeding values resulted among different models. That would indicate that a few test-days per cow would produce accurate breeding values for milkability.

Key words: milking system, milk flow rate, genetic evaluation, genetic parameter

\section{INTRODUCTION}

In recent years, interest has increased in functional traits in dairy cows because of their effect on profitability through cost-saving opportunities. One such functional trait is milkability. In modern milking systems, slow-milking cows may hold up the milking process when many cows are milked together or lead to less efficient use of milking robots. Use of modern milking systems is rapidly increasing: in Sweden, almost $30 \%$ of cows are milked in automatic (voluntary) milking systems (AMS), about $25 \%$ in conventional milking parlors (CMP), and about $45 \%$ are milked in stanchion barns (spring of 2013; N.-E. Larsson, Växa Sweden, Stockholm, Sweden, personal communication). Selection against slow-milking cows would be of interest to save time and to make efficient use of expensive equipment.

Modern milking systems offer opportunities for inline recording of several milkability traits. Carlström et al. (2013) showed the value of using AMS data from commercial herds for genetic analysis. In-line collected milkability data required strict editing before analysis but then showed high repeatabilities $(0.85-0.88)$ and moderately high heritabilities $(0.36-0.51)$ for average milk flow rate (AFR) and box time (BT) in different lactations of the 2 major Swedish dairy breeds, Swedish Holstein (SH) and Swedish Red (SR). These estimates are comparable with results from studies on heritability for milkability in AMS in research herds (Gäde et al., 2006; Løvendahl et al., 2011). In the AMS study by Carlström et al. (2013), milking interval and milking frequency were also recorded, but they were not found as suitable as AFR and BT for genetic analysis. In regard to milkability recorded in CMP, a Swedish pilot 
study by Norgren (2005) noted the potential for using such data, but low heritability estimates were shown for flow rate as well as for milking time. Those results clearly indicated the need for better understanding of data collection, data editing, and the use of in-line recorded milkability data from milking parlors for genetic analysis purposes.

Since 2007 in Sweden, dairy farmers using CMP, as well as management software that is compatible with the national milk recording scheme, have been able to share milkability information together with the regular test-day milking observations. However, routine genetic evaluations for milkability traits do not yet consider these data. With the rapid development toward the use of milking systems offering in-line recording of milkability traits, the issue is how to make best use of such data. Are conventional parlor data, which are less extensive, as valuable for genetic analyses as AMS data? Laureano et al. (2012) studied monthly objective milkability data from first-lactation Holstein cows in a CMP system and found heritabilities in the range of 0.23 to 0.37 depending on the model used. High genetic correlations between monthly observations indicated that direct selection for one test-day may result in genetic gain of milkability during the whole lactation. Their results suggested that less extensive CMP data would be highly valuable for genetic evaluation purposes. However, results were based on research farm data and not on commercial herds. Furthermore, in AMS herds, cows are milked on a voluntary basis, usually 2 to 4 times a day, whereas CMP data come from a system with strict milking intervals for all cows, which are usually milked twice a day. Thus, AMS and CMP represent different systems of handling cows at milking. Data are captured automatically in both systems but the traits are differently defined and measured in the 2 systems.

The overall objective of the current study was to investigate if data from AMS and CMP can be jointly used for genetic evaluation. The specific objectives were (1) to estimate genetic parameters for milkability data solely from commercial herds with CMP, (2) to estimate genetic correlations for different milkability traits across milking systems; that is, CMP and AMS, and (3) to find a suitable model to analyze data from the 2 different milking systems for genetic evaluation purposes. To our knowledge, no previous study has included analyses of joint observations from both CMP and AMS.

\section{MATERIALS AND METHODS}

\section{Data from CMP Systems}

Milkability data from CMP were extracted from the national milk recording scheme run by Växa Sweden
(Stockholm). Herds with milking units that automatically record milkability as milking time; that is, the time from start to end of the milking (MT) and milk yield (MY) per milking, may deliver this information to the milk recording scheme once a month together with the regular test-day milking observations. At each test-day, milkability information for all milkings from the previous $48 \mathrm{~h}$ are sent to the milk recording scheme, resulting in up to 6 , with an average of 3 , observations per cow and month.

The original data set included all available milkability information recorded from the start of this service in March 2007 to March 2011. Data comprised information from 74 herds. Complementary information, to connect each cow to a pedigree and each milkability observation to a parity number and lactation stage, was also provided by Växa Sweden. After merging the milkability data with information about pedigree, lactation number, and stage of lactation, the data were edited. Observations from parity 4 or higher were discarded as were DIM earlier than d 8 or later than d 330 of the lactation. The remaining data set comprised 483,437 observations from 18,786 cows. Ten herds contributed with observations only from SH, 2 herds had only SR cows, whereas 62 herds contributed with records of both breeds.

The AFR was calculated in kilograms per minute as the ratio between MY and MT (Table 1). Observations that were clearly inconsistent with normal variation regarding MY, MT, or AFR were excluded from the analysis. Observations with MY $<2 \mathrm{~kg}$ or $>35 \mathrm{~kg}$ at a single milking were excluded, as were MT $<1$ min. Observations of MT and AFR were excluded if they exceeded more than 3 standard deviations from the mean of the respective breed. With these restrictions, 1.6 and $2.0 \%$ of the observations for $\mathrm{SH}$ and SR, respectively, were discarded.

\section{Data from AMS}

Milkability data recorded from 2004 to 2009 in herds with AMS were provided for 19 dairy herds across Sweden, using DeLaval automatic milking systems (DeLaval Inc., Tumba, Sweden). The milkability traits recorded in the AMS or calculated on the basis of recorded variables were AFR, BT, and MT. Complementary information to connect each cow to a pedigree and each milkability observation to a parity number and lactation stage was done as for the CMP data. Only observations from the first 3 lactations and from DIM 8 to 330 within each lactation were kept. The remaining data set included 3,377,231 observations from 4,080 cows. A thorough editing process was needed for the AMS data, with restrictions regarding what should be 
interpreted as normal milkings (i.e., observations classified as complete by the AMS system dependent on milk yield in each udder quarter compared with previous milkings and where no removal of teat cups from any udder quarter was recorded), as well as exclusion of records clearly inconsistent with normal variation of the traits. The data set with milkability information from AMS and its editing was described in detail by Carlström et al. (2013).

\section{Data Structure}

The final CMP data set included 271,638 observations from 11,123 $\mathrm{SH}$ cows and 203,117 observations from 7,554 SR cows. The final AMS data set included $1,063,951$ observations from $1,053 \mathrm{SH}$ cows and $1,155,732$ observations from 1,749 SR cows. Loss of observations in the final data sets, due to the editing criteria described above, was much greater for the AMS data set $(34 \% ; 6.8 \%$ loss of cows) than for the data from CMP $(1.8 \% ; 0.4 \%$ loss of cows), which reflects the fact that AMS saves information about every milking from each cow regardless of the quality of data or milking.

Pedigree information included 3 generations of ancestors. In total, 8,194 and 36,307 animals were included in the pedigree file for $\mathrm{SH}$ in AMS and CMP, respectively. Correspondingly, 6,519 and 25,116 animals were included in the pedigree file for SR. In AMS, the SH (SR) cows with observations were daughters of 421 (340) sires, where $24 \%$ of the SH sires and $16 \%$ of the SR sires had at least 5 daughters. The SH (SR) cows in CMP were daughters of 977 (681) sires, where $41 \%$ of the SH sires and $35 \%$ of the SR sires had at least 5 daughters. For SH (SR), 68\% (75\%) of the sires with at least 5 daughters in the AMS data also had at least as many daughters with milkability observations in the conventional parlor system.

\section{Estimation of (Co)Variance Components and Model Comparisons}

In the present study, the initial analysis to estimate variance components, heritabilities, repeatability coefficients, and permanent environmental effects for the CMP data was performed in the same way as was previously done for AMS data by Carlström et al. (2013), using a repeatability model including the random effects of $p e, a$, and $e$, where pe is the random permanent environmental effect of cow to account for repeated measurements within cow: $\sim N\left(0, \mathbf{I} \sigma_{p e}^{2}\right)$, where $N$ is normally distributed, $\mathbf{I}$ is an identity matrix, and $\sigma_{p e}^{2}$ is the permanent environment variance; $a$ is the random additive genetic effect of cow: $\sim N\left(0, \mathbf{A} \sigma_{a}^{2}\right)$, where $\mathbf{A}$ is the 
additive genetic relationship matrix, and $\sigma_{a}^{2}$ is genetic effects variance; and $e$ is the random residual effect belonging to the observation $\sim N\left(0, \sigma_{e}^{2}\right)$, where $\sigma_{e}^{2}$ is the residual error variance. Covariances between all random effects ( $a$, pe, and $e$ ) were assumed to be zero (Model [1]; Table 2).

The 2 breeds were analyzed separately. Milkability traits in first and later (second and third) lactations were, in this first analysis, considered to be different traits and therefore analyzed univariately. Model [2] (Table 2) was used for the analyses of later lactations, where the fixed effect of lactation number included 2 levels ( 2 and 3$)$, and $p e$ is now also across lactations.

In the next steps of analysis, we estimated genetic correlations between different milkability traits as well as between lactations and between the same trait measured in different milking systems. Genetic correlations between traits and between lactations (first versus later), within milking system (CMP and AMS) were estimated in a bivariate setting using the appropriate combination of Models [1] and [2].

The (co)variance structure among vectors of random variables in the bivariate models with 2 traits in the same lactation was

$\operatorname{var}\left[\begin{array}{l}p e_{1} \\ p e_{2} \\ a_{1} \\ a_{2} \\ e_{1} \\ e_{2}\end{array}\right]=\left[\begin{array}{cccccc}\mathbf{I} \sigma_{p e_{1}}^{2} & \mathbf{I} \sigma_{p e_{1} p e_{2}} & 0 & 0 & 0 & 0 \\ \mathbf{I} \sigma_{p e_{1} p e_{2}} & \mathbf{I} \sigma_{p e_{2}}^{2} & 0 & 0 & 0 & 0 \\ 0 & 0 & \mathbf{A} \sigma_{a_{1}}^{2} & \mathbf{A} \sigma_{a_{12}} & 0 & 0 \\ 0 & 0 & \mathbf{A} \sigma_{a_{12}} & \mathbf{A} \sigma_{a_{2}}^{2} & 0 & 0 \\ 0 & 0 & 0 & 0 & \mathbf{I} \sigma_{e_{1}}^{2} & \mathbf{I} \sigma_{e_{12}} \\ 0 & 0 & 0 & 0 & \mathbf{I} \sigma_{e_{12}} & \mathbf{I} \sigma_{e_{2}}^{2}\end{array}\right]$,

and for the bivariate models including the same traits in different lactations:

$\operatorname{var}\left[\begin{array}{l}p e_{1} \\ p e_{2} \\ a_{1} \\ a_{2} \\ e_{1} \\ e_{2}\end{array}\right]=\left[\begin{array}{cccccc}\mathbf{I} \sigma_{p e_{1}}^{2} & \mathbf{I} \sigma_{p e_{1} p e_{2}} & 0 & 0 & 0 & 0 \\ \mathbf{I} \sigma_{p e_{1} p e_{2}} & \mathbf{I} \sigma_{p e_{2}}^{2} & 0 & 0 & 0 & 0 \\ 0 & 0 & \mathbf{A} \sigma_{a_{1}}^{2} & \mathbf{A} \sigma_{a_{12}} & 0 & 0 \\ 0 & 0 & \mathbf{A} \sigma_{a_{12}} & \mathbf{A} \sigma_{a_{2}}^{2} & 0 & 0 \\ 0 & 0 & 0 & 0 & \mathbf{I} \sigma_{e_{1}}^{2} & 0 \\ 0 & 0 & 0 & 0 & 0 & \mathbf{I} \sigma_{e_{2}}^{2}\end{array}\right]$.

For the bivariate models including the same traits in different lactations, the residual correlations pertaining to observations do not exist because the same milking cannot exist both in lactation 1 and lactations $2+3$.

Genetic relationships between traits across milking systems were analyzed, where AFR and MT measured in different milking systems were considered to be different traits. Based on the results from the previous bivariate analysis (Model [1] and [2]), the milkability traits in different lactations could be considered as the same trait. Model [2] (Table 2) was used and the fixed effect of lactation number now included 3 levels (1-3). The (co)variance structure among vectors of random variables in this bivariate model was

$$
\operatorname{var}\left[\begin{array}{l}
p e_{1} \\
p e_{2} \\
a_{1} \\
a_{2} \\
e_{1} \\
e_{2}
\end{array}\right]=\left[\begin{array}{cccccc}
\mathbf{I} \sigma_{p e_{1}}^{2} & 0 & 0 & 0 & 0 & 0 \\
0 & \mathbf{I} \sigma_{p e_{2}}^{2} & 0 & 0 & 0 & 0 \\
0 & 0 & \mathbf{A} \sigma_{a_{1}}^{2} & \mathbf{A} \sigma_{a_{12}} & 0 & 0 \\
0 & 0 & \mathbf{A} \sigma_{a_{12}} & \mathbf{A} \sigma_{a_{2}}^{2} & 0 & 0 \\
0 & 0 & 0 & 0 & \mathbf{I} \sigma_{e_{1}}^{2} & 0 \\
0 & 0 & 0 & 0 & 0 & \mathbf{I} \sigma_{e_{2}}^{2}
\end{array}\right] .[3]
$$

For the bivariate models including the same traits in different milking systems, neither the permanent environment correlations pertaining to cow nor the residual correlations pertaining to observations exist, because each cow only appears in AMS or CMP.

Finally, based on the results from bivariate analysis of Model [2], with different milking systems, all data from both kinds of systems could be included in the same univariate analysis assuming that respective traits in the 2 milking systems are the same ones (Model [3]; Table 2). Because the variance differed between systems and lactations, the data were preadjusted for heterogeneous variance between AMS and CMP, as well as between lactations. Standard deviations for the CMP data are presented in Table 1 and corresponding values for the AMS data were presented by Carlström et al. (2013). Lactation 1 in CMP was used as the reference:

$$
y^{*}=\left[\left(y-\bar{y}_{\text {subgroup }}\right) \times \sqrt{\operatorname{var}_{C M P, \text { lact } 1} / \operatorname{var}_{\text {subgroup }}}\right]+\bar{y}_{\text {subgroup }},
$$

where $y^{*}$ is the milkability observation adjusted for heterogeneous variance; $y$ is the original milkability observation; $\bar{y}_{\text {subgroup }}$ is the mean value of the milkability observations in the group of $y$; for example, observations from first-lactation cows in AMS; $\operatorname{var}_{C M P \text {,lact } 1}$ is the variance of the observations in the reference group; and $\operatorname{var}_{\text {subgroup }}$ is the variance of the observations in the group of $y$. 
To be able to better compare with the results from the random regression analyses (see below), Model [3] was also applied to data between DIM 31 and 250 only.

One aim of this study was to find a suitable model for genetic evaluation purposes. At this point, different models were therefore compared through model fit based on the log-likelihood function and on error variance. The models under consideration were the repeatability model, Model [3], and 4 different random regression (RR) models. Model [3] was then extended by adding first-, second-, third-, or fourth-order Legendre polynomials of DIM for the random effects $a$ and pe, resulting in the $\mathrm{RR}$ models denoted RR1 to RR4, respectively (RRq model; Table 2 ).

(Co)variance components for all models were estimated using average information (AI)-REML (Jensen et al., 1997), included in the DMU package of Madsen and Jensen (2007). The convergence criterion was chosen so that the norm of the update vector for the (co) variance components was $<10^{-7}$ and the norm of the gradient vector (AI) was $<10^{-6}$. Some of the bivariate analyses did not converge with this strict convergence criterion, so it was reset to $10^{-5}$ to obtain estimates and standard errors. For the RRq model, at DIM $p, \sigma_{a_{p}}^{2}$ and $\sigma_{p e_{p}}^{2}$ were calculated as

$$
\begin{gathered}
\sigma_{a_{p}}^{2}=\mathbf{z}_{p}^{\prime} \mathbf{G} \mathbf{z}_{p}, \\
\sigma_{p e_{p}}^{2}=\mathbf{z}_{p}^{\prime} \mathbf{P} \mathbf{z}_{p},
\end{gathered}
$$

where $\mathbf{z}_{p}$ is a vector of polynomials in the model for DIM $p, \mathbf{G}$ is the $(\mathrm{q}+1) \times(\mathrm{q}+1)(\mathrm{co})$ variance matrix for animal RR coefficients, and $\mathbf{P}$ is the (co)variance matrix for permanent environment RR coefficients.

The heritabilities were calculated as $\sigma_{a}^{2} / \sigma_{p}^{2}$, where $\sigma_{p}^{2}=\sigma_{p e}^{2}+\sigma_{a}^{2}+\sigma_{e}^{2}$. The proportion of variance due to the permanent environmental effect to the total phenotypic variance $\left(c^{2}\right)$ was calculated as $\sigma_{p e}^{2} / \sigma_{p}^{2}$, and the repeatabilities $(t)$ across all milkings were calculated as $\left(\sigma_{p e}^{2}+\sigma_{a}^{2}\right) / \sigma_{p}^{2}$, all parameters being a function of DIM.

Estimated breeding values of all cows with milkability records for AFR were obtained from the repeatability model (Model [3]) and from the RR3 model. Pearson correlations of cow EBV from Model [3] with cow EBV from RR3 model were computed.

\section{RESULTS AND DISCUSSION}

\section{Genetic Variation in CMP Data}

The heritability estimates from Models [1] and [2] were of the same magnitude for both breeds and ranged from moderate to high (0.27-0.43; Table 3$)$. For both AFR and MT, the heritabilities tended to decrease from first to later lactations. Estimated heritabilities were comparable with those reported by Carlström et al. (2013) and Byskov et al. (2012).

The repeatability coefficients were high and similar between breeds and traits, ranging from 0.63 to 0.77 in first lactation and from 0.63 to 0.70 in later lactations. These results are comparable with Laureano et al. (2012), who estimated a coefficient of repeatability for AFR of 0.66 based on objective observations in a

Table 2. Description of models used for the genetic analyses in conventional milking parlors (CMP) and automatic milking systems (AMS), where $\mathrm{y}$ is the analyzed milkability trait, average flow rate, milking time, or box time

\begin{tabular}{lll}
\hline Model name & Milking system & Model description \\
\hline Model $1^{1}$ & CMP & $y_{i j k l}=\mu+H Y S_{i}+L M_{j}+p e_{k}+a_{k}+\beta_{1} M Y_{i j k l}+e_{i j k l}$ \\
Model $2^{2}$ & CMP or AMS & $y_{i j k l m}=\mu+H Y S_{i}+L M_{j}+L_{l}+p e_{k}+a_{k}+\beta_{1} M Y_{i j k l m}+e_{i j k l m}$ \\
Model $3^{3}$ & CMP and AMS & $y_{i j k l m n}=\mu+H Y S_{i}+L M_{j}+L_{l}+S_{m}+p e_{k}+a_{k}+\beta_{m} M Y(S)_{i j k l m n}+e_{i j k l m n}$ \\
RRq models $^{4}$ & CMP and AMS & $y_{i j k l m n p}=\mu+H Y S_{i}+L M_{j}+L_{l}+S_{m}+\beta_{m} M Y(S)_{i j k l m n}+\sum_{o=0}^{q} a_{k o} Z_{p o}+\sum_{o=0}^{q} p e_{k o} Z_{p o}+e_{i j k l m n p}$ \\
\hline
\end{tabular}

\footnotetext{
${ }^{1}$ Where $\mu$ is the overall mean; $H Y S_{i}$ is the fixed effect of ith combination of herd, with year and season of observation (January-April, MayAugust, September-December); $L M_{j}$ is the fixed effect of $j$ th lactation month; $p e_{k}$ is the random permanent environmental effect of cow $k$ to account for repeated measurements within cow, $\sim N\left(0, \mathbf{I} \sigma_{p e}^{2}\right)$; where $N$ is normally distributed, $\mathbf{I}$ is an identity matrix, and $\sigma_{p e}^{2}$ is the permanent environment variance; $a_{k}$ is the random additive genetic effect of cow $k, \sim N\left(0, \mathbf{A} \sigma_{a}^{2}\right)$, where $\mathbf{A}$ is the additive genetic relationship matrix, and $\sigma_{a}^{2}$ is genetic effects variance; $\beta_{1} M Y_{i j k l}$ is the effect of milk yield for the particular milking $l$ of cow $k$ in $H Y S_{i}$ and $L M_{j}$ as a fixed linear covariate; and $e_{i j k l}$ is the random residual effect belonging to observation $y_{i j k l} \sim N\left(0, \mathbf{I} \sigma_{e}^{2}\right)$, where $\sigma_{e}^{2}$ is the residual error variance.

${ }^{2}$ Additional effects to those included in Model [1]: $L_{l}$ is the fixed effect of $l$ th lactation number.

${ }^{3}$ Additional effects to those included in Model [2]: $S_{m}$ is the fixed effect of $m$ th milking system; and $\beta_{m} M Y(S)_{i j k l m n}$ is the effect of $M Y$ for the particular milking $n$ of cow $k$ in $H Y S_{i}, L M_{j}, L_{l}$, and $S_{m}$ as a fixed linear covariate nested within $S$, resulting in 2 regression coefficients.

${ }^{4} \mathrm{RRq}=$ random regression models with q order Legendre polynomials of DIM for the permanent environmental and animal effects. Additional effects to those included in Model [3]: $Z_{p o}$, the polynomial $o$ for DIM $p$, where $o=0,1,2,3,4$ (same order for both the permanent environmental and animal effects); $a_{k o}$ is the random regression coefficient on $Z_{p o}$ for the animal genetic effect; and $p e_{k o}$ is the random regression coefficient on $Z_{p o}$ for the permanent environmental effect.
} 
Table 3. Genetic $\left(\sigma_{a}^{2}\right)$, permanent environment $\left(\sigma_{p e}^{2}\right)$, and random residual $\left(\sigma_{e}^{2}\right)$ variance components and heritabilities $\left(h^{2}\right)$ for Swedish Holstein (SH) and Swedish Red (SR) in conventional milking parlors (SE in parentheses)

\begin{tabular}{llcccc}
\hline $\begin{array}{l}\text { Breed } \\
\text { and trait }\end{array}$ & $\begin{array}{l}\text { Lactation } \\
\text { number }\end{array}$ & $\sigma_{a}^{2}$ & $\sigma_{p e}^{2}$ & $\sigma_{e}^{2}$ & $h^{2}$ \\
\hline SH & & & & & \\
AFR & 1 & $0.13(0.01)$ & $0.14(0.01)$ & $0.09(<0.01)$ & $0.37(0.04)$ \\
& $2+3$ & $0.09(0.01)$ & $0.14(0.01)$ & $0.12(<0.01)$ & $0.27(0.03)$ \\
MT & 1 & $0.85(0.08)$ & $0.72(0.06)$ & $0.47(<0.01)$ & $0.42(0.04)$ \\
SR & $2+3$ & $0.60(0.07)$ & $0.68(0.05)$ & $0.55(<0.01)$ & $0.33(0.03)$ \\
AFR & 1 & $0.10(0.01)$ & $0.09(0.01)$ & $0.07(<0.01)$ & $0.38(0.04)$ \\
& $2+3$ & $0.07(0.01)$ & $0.08(0.01)$ & $0.09(<0.01)$ & $0.30(0.04)$ \\
MT & 1 & $0.78(0.09)$ & $0.58(0.07)$ & $0.45(<0.01)$ & $0.43(0.05)$ \\
& $2+3$ & $0.52(0.07)$ & $0.54(0.05)$ & $0.54(<0.01)$ & $0.33(0.04)$ \\
\hline
\end{tabular}

${ }^{1} \mathrm{AFR}=$ average milk flow rate; $\mathrm{MT}=$ milking time.

conventional milking parlor system. The present results were slightly lower than the repeatabilities of 0.85 to 0.89 for AFR estimated by Carlström et al. (2013) based on observations from AMS. The results from the AMS study were based on daily observations, whereas the present study only included observations from 1 test round, consisting of milkings from 2 consecutive days per month, which could explain the difference. First-lactation cows had, on average, 17 and 18 observations per cow for $\mathrm{SH}$ and SR, respectively, coming from, on average, 5 and 6 test rounds. Even with this decreased amount of information per cow compared with our previous AMS study, where first-lactation cows averaged 340 and 392 observations per cow for $\mathrm{SH}$ and SR, respectively, the repeatability coefficients were high. This result indicated that 1 test-day would guarantee accurate milkability information, which was also indicated by Carlström et al. (2013) for AMS data.

The permanent environmental variation was large for both traits in first and in later lactations. Proportion of permanent environmental variation of total phenotypic variation, $c^{2}$, was the same size as the heritability, with $c^{2}$ estimates of 0.32 to 0.39 .

\section{Genetic Correlations Between Lactations, Traits, and Milking Systems}

The genetic relationships for AFR and MT across parities were high and at the same level for both breeds in CMP (0.97-0.98) and in AMS data (0.93-0.99). The same was true for relationships with BT in AMS (0.94-1.00). Standard errors for the estimates of genetic correlations were in the range of 0.01 to 0.03 . The results from the present study agree well with Gäde et al. (2007), who showed genetic correlations in the range of 0.88 to 0.98 for AFR, MT, and maximum flow rate between the first 3 lactations with data from conventional milking parlor systems. Dodenhoff and Emmer- ling (2009) divided each of the first 3 lactations into 6 periods and estimated genetic correlations in the range of 0.65 to 0.99 for AFR between the different periods within and between lactations. The high genetic correlations estimated in the present study indicate that it is not necessary to consider milkability as different traits in different lactations. Instead of using multipletrait models for genetic analyses, it should be sufficient to use a repeatability model where a fixed effect of lactation number is included.

This study clearly showed that milkability measured as AFR, MT, or BT was highly genetically correlated in both breeds. In AMS, negative genetic correlations of -0.93 to -1.00 were found between AFR and the traits representing time for the milking process (MT and $\mathrm{BT}$ ), whereas we found high positive genetic correlations between MT and BT (0.93-1.00). In CMP, the corresponding genetic correlations between AFR and MT were -0.94 to -0.99 . Standard errors for the estimates of genetic correlations were in the range of 0.01 to 0.04 . High genetic correlations between AFR and the time traits were expected as flow rate is a function of time. The estimates from the present study are even higher than those reported by other authors. Gray et al. (2011) estimated a genetic correlation of -0.90 between AFR and MT in first lactation. Gäde et al. (2007) reported a genetic correlation of -0.77 between AFR and MT when milkability was measured in conventional milking parlors, and a corresponding correlation of -0.90 when milkability observations came from AMS. The stronger estimated genetic correlations in the present study could be due to the fact that much more data, in total 22,479 cows of 73 herds, were included. Gray et al. (2011) had only 1 observation per cow in first lactation. The results in Gäde et al. (2006) were based on observations from only 1 research AMS herd, whereas only 2 conventional parlor herds were included in Gäde et al. (2007). 
The high estimated genetic correlations between BT and MT in AMS indicate that it is not necessary to use both traits. We suggest that BT is the better alternative for 2 reasons. First, the total time each cow spends in the milking box is a very important economic parameter for AMS herds, in order to serve as many cows as possible per box. Second, BT is measured directly in the AMS based on registered times for when the cow enters and leaves the milking box, whereas MT is indirectly calculated based on records of milk yield and AFR.

As AMS and CMP constitute 2 different systems of handling cows at milking - one voluntary and one strictly regulated milking system - it is of interest to determine if data from the 2 systems could be used jointly for genetic evaluations. Furthermore, methods for recording, defining traits, and storing milkability data differ between milking equipment companies and management software programs. If the genetic correlations between milkability traits recorded in different systems are high, it should be possible to treat them as the same trait in genetic evaluations. The genetic correlations estimated with Model [3] between AFR, as well as MT (BT) measured in AMS and in CMP, were high (0.93-1.00) for both breeds (Table 4). Even though AFR was measured directly per udder quarter in AMS and calculated based on measurements of total milking time and milk yield in CMP, the correlations were close to unity. The same was true for MT, which was measured directly in the CMP, whereas it was calculated based on measurements of milk yield and AFR in AMS. Therefore, we can assume that it is the same trait measured in 2 different systems, although these systems differ substantially and data are recorded differently. Byskov et al. (2012) estimated the genetic correlation between milk fat and protein flow rates measured in the Lely AMS (Lely, Maassluis, the Netherlands), and milk fat and protein flow rates measured by TruTest milk meters (TruTest Ltd., Auckland, New Zealand) in non-AMS, in first-lactation Danish Holstein cows. Their estimated genetic correlation of 0.94 is in agreement with the results in our study.

\section{Model Comparisons for Genetic Evaluation}

For all traits, a higher order of RR model resulted in lower error variance and $-2 \operatorname{LogL}$ estimates (Table 5). This trend was also seen when RR4 was used (results not shown in table) but the decrease in the model fit parameters was smaller and the analyses did not converge for all traits, and thus, we decided that order 3 of Legendre polynomials was sufficient.

In Table 6 , the lactations are split into 3 periods: DIM 8-30, DIM 31-250, and DIM 251-330. In the RR
Table 4. Genetic correlations between automatic (AMS) and conventional (CMP) milking system traits ${ }^{1}$ from Model [3], including all lactations (SE in parentheses) ${ }^{2}$

\begin{tabular}{llrr}
\hline $\begin{array}{l}\text { Trait } \\
\text { in AMS }\end{array}$ & Trait in & \multicolumn{1}{c}{ SH } & \multicolumn{1}{c}{ SR } \\
\hline AFR & AFR & $0.97(0.04)$ & $0.98(0.03)$ \\
AFR & MT & $-0.96(0.04)$ & $-0.99(0.03)$ \\
MT & AFR & $-1.00(0.03)$ & $-0.95(0.04)$ \\
MT & MT & $0.98(0.04)$ & $1.00(0.04)$ \\
BT & AFR & $-0.98(0.04)$ & $-0.94(0.04)$ \\
BT & MT & $0.99(0.03)$ & $0.93(0.05)$ \\
\hline
\end{tabular}

${ }^{1} \mathrm{AFR}=$ average flow rate; $\mathrm{MT}=$ milking time; $\mathrm{BT}=$ box time.

${ }^{2} \mathrm{SH}=$ Swedish Holstein; SR $=$ Swedish Red.

analyses, variance components were estimated for each DIM. Estimates from RR analyses shown in Table 6 for heritability, additive genetic variation, and permanent environmental variation as a proportion of the phenotypic variation are average values for all DIM included in respective period. Heritability estimates from Model [3] were 0.44 to $0.49,0.38$ to 0.41 , and 0.24 to 0.43 for AFR, MT, and BT, respectively, based on observations from period 2, and similar for the RR models. Heritability estimates from Model [3], based on observations from the total lactation (DIM 8-330), are shown in parentheses in Table 6 . The heritability estimates are in the same range as, or slightly higher than, those reported by other authors for milkability studies based on objective measurements in CMP (e.g., Rensing and Ruten, 2005; Dodenhoff and Emmerling, 2009; Laureano et al., 2012). However, the comparison of results from different studies is difficult because of the use of different numbers of observations where only one observation is included in some studies, and monthly, weekly, or daily repeated observations are included in others. The use of different definitions of traits and statistical models further complicates comparisons.

The results from the repeatability model showed that the repeatability coefficients were almost identical between breeds, ranging from 0.69 to 0.80 , with the lowest values for MT and the highest for AFR. Estimated trajectories of heritability over the lactation for the 3 milkability traits analyzed with the repeatability Model [3] and the RR3 model are shown in Figure 1. The pattern of the lines from the RR3 model indicates that heritability, for AFR and MT in particular, is constant during the main part of the lactation but lower in the beginning and late in lactation (after DIM 300). Even though this could be due to statistical constraints depending on a limited number of observations from the extreme ends of the lactation, this could be important to consider when deciding which observations should be collected for genetic evaluation purposes. Figure 1 shows the heritability estimates from Model [3] based on observations from DIM 31 to 250 only as well as 
Table 5. Estimates of error variance $\left(\sigma_{e}^{2}\right)$ and $\log$-likelihood function $(-2 \mathrm{LogL})$ for average flow rate (AFR), milking time (MT), and box time (BT) from a repeatability model (Rep, Model [3]) and random regression models with second-order (RR2) and third-order (RR3) Legendre polynomials for additive genetic and permanent environmental effects

\begin{tabular}{|c|c|c|c|c|c|c|c|c|c|c|c|c|}
\hline \multirow[b]{3}{*}{ Trait } & \multicolumn{6}{|c|}{ Swedish Holstein } & \multicolumn{6}{|c|}{ Swedish Red } \\
\hline & \multicolumn{3}{|c|}{$\sigma_{e}^{2}$} & \multicolumn{3}{|c|}{$-2 \log \mathrm{L}$} & \multicolumn{3}{|c|}{$\sigma_{e}^{2}$} & \multicolumn{3}{|c|}{$-2 \log \mathrm{L}$} \\
\hline & Rep & $\mathrm{RR} 2$ & RR3 & Rep & $\mathrm{RR} 2$ & RR3 & Rep & $\mathrm{RR} 2$ & RR3 & Rep & $\mathrm{RR} 2$ & RR3 \\
\hline AFR & 0.068 & 0.055 & 0.053 & $-215,262$ & $-239,544$ & $-243,214$ & 0.063 & 0.051 & 0.049 & $-230,281$ & $-256,250$ & $-259,383$ \\
\hline MT & 0.690 & 0.554 & 0.533 & 880,633 & 627,978 & 587,143 & 0.400 & 0.330 & 0.321 & 154,160 & $-69,786$ & $-99,806$ \\
\hline BT & 1.176 & 1.009 & 0.982 & $1,250,931$ & $1,102,214$ & $1,077,150$ & 1.201 & 1.033 & 1.010 & $1,380,666$ & $1,219,811$ & $1,197,410$ \\
\hline
\end{tabular}

Table 6. Additive genetic variance components $\left(\sigma_{a}^{2}\right)$, proportion of permanent environmental variance to phenotypic variance $\left(c^{2}\right)$ and heritabilities $\left(h^{2}\right)$ from a repeatability model (Rep, Model [3] for period 2) and random regression models with second-order (RR2) and third-order (RR3) Legendre polynomials for additive genetic and permanent environmental effects

\begin{tabular}{|c|c|c|c|c|c|c|c|c|c|c|c|c|c|c|c|c|c|c|c|}
\hline \multirow[b]{3}{*}{ Trait $^{1}$} & \multirow[b]{3}{*}{ Period $^{2}$} & \multicolumn{9}{|c|}{ Swedish Holstein } & \multicolumn{9}{|c|}{ Swedish Red } \\
\hline & & \multicolumn{3}{|c|}{$\sigma_{a}^{2}$} & \multicolumn{3}{|c|}{$c^{2}$} & \multicolumn{3}{|c|}{$h^{2}$} & \multicolumn{3}{|c|}{$\sigma_{a}^{2}$} & \multicolumn{3}{|c|}{$c^{2}$} & \multicolumn{3}{|c|}{$h^{2}$} \\
\hline & & Rep & RR2 & RR3 & Rep & $\mathrm{RR} 2$ & RR3 & Rep & $\mathrm{RR} 2$ & RR3 & Rep & $\mathrm{RR} 2$ & RR3 & Rep & $\mathrm{RR} 2$ & RR3 & Rep & $\mathrm{RR} 2$ & RR3 \\
\hline \multirow[t]{3}{*}{ AFR } & 1 & & 0.15 & 0.15 & & 0.43 & 0.42 & & 0.42 & 0.43 & & 0.11 & 0.11 & & 0.40 & 0.40 & & 0.41 & 0.41 \\
\hline & 2 & 0.19 & 0.21 & 0.21 & 0.34 & 0.32 & 0.32 & 0.49 & 0.54 & 0.54 & 0.11 & 0.12 & 0.12 & 0.34 & 0.36 & 0.36 & 0.44 & 0.45 & 0.45 \\
\hline & 3 & $(0.18)^{3}$ & 0.21 & 0.20 & $(0.33)$ & 0.34 & 0.36 & $(0.49)$ & 0.52 & 0.51 & $(0.11)$ & 0.10 & 0.10 & $(0.32)$ & 0.42 & 0.42 & $(0.43)$ & 0.39 & 0.39 \\
\hline \multirow[t]{3}{*}{ MT } & 1 & & 0.97 & 0.94 & & 0.45 & 0.44 & & 0.35 & 0.36 & & 0.82 & 0.80 & & 0.41 & 0.41 & & 0.42 & 0.42 \\
\hline & 2 & 0.93 & 0.99 & 1.00 & 0.36 & 0.37 & 0.36 & 0.38 & 0.40 & 0.42 & 0.69 & 0.73 & 0.76 & 0.36 & 0.37 & 0.27 & 0.41 & 0.43 & 0.44 \\
\hline & 3 & $(0.85)$ & 0.69 & 0.73 & $(0.34)$ & 0.35 & 0.38 & $(0.36)$ & 0.36 & 0.36 & $(0.65)$ & 0.46 & 0.52 & $(0.35)$ & 0.45 & 0.44 & $(0.40)$ & 0.32 & 0.34 \\
\hline \multirow[t]{3}{*}{ BT } & 1 & & 1.63 & 1.41 & & 0.47 & 0.48 & & 0.32 & 0.31 & & 2.32 & 2.22 & & 0.36 & 0.34 & & 0.44 & 0.46 \\
\hline & 2 & 1.01 & 1.10 & 1.18 & 0.49 & 0.50 & 0.48 & 0.24 & 0.26 & 0.28 & 1.86 & 1.96 & 2.18 & 0.31 & 0.32 & 0.28 & 0.43 & 0.44 & 0.49 \\
\hline & 3 & $(0.96)$ & 0.82 & 0.89 & $(0.46)$ & 0.45 & 0.46 & $(0.24)$ & 0.25 & 0.25 & $(1.90)$ & 1.11 & 1.28 & $(0.27)$ & 0.45 & 0.45 & $(0.45)$ & 0.29 & 0.31 \\
\hline
\end{tabular}

${ }^{1}$ For average flow rate (AFR) and milking time (MT), observations were from both automatic and conventional milking parlors, whereas observations of box time (BT) were only from automatic milking systems.

${ }^{2}$ Period 1: DIM 8-30; period 2: DIM 31-250; period 3: DIM 251-330.

${ }^{3}$ Estimates from the repeatability model for the total lactation (DIM 8-330) are given in parentheses. 

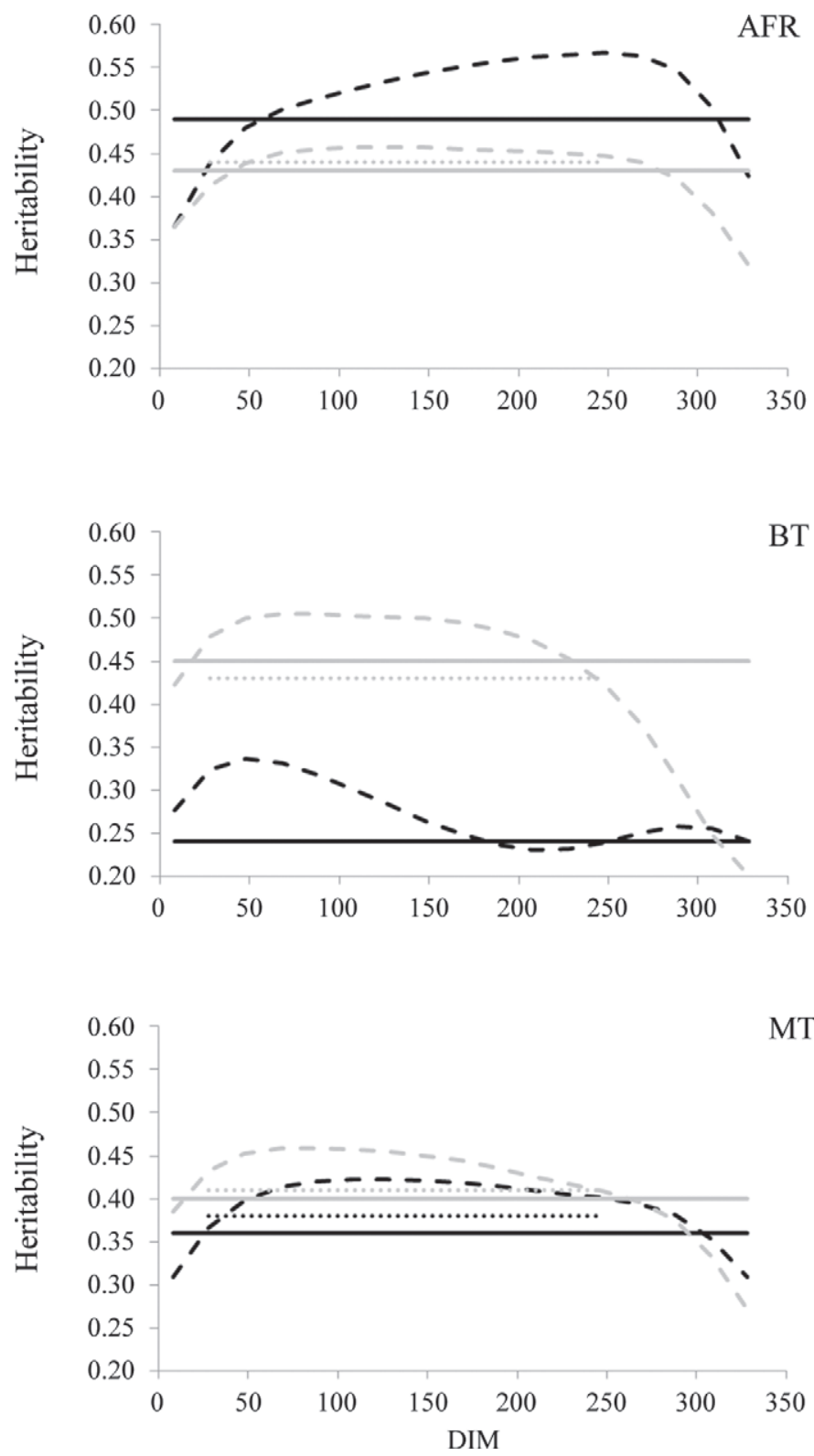

Figure 1. Estimated trajectories of heritability ( $h^{2}$; dashed lines) over DIM for average flow rate (AFR), milking time (MT), and box time (BT) from a random regression model with third-order Legendre polynomials for additive genetic and permanent environmental effects. Heritability from a repeatability model (Model [3]) are indicated with a horizontal dotted line for DIM 31 to 250 and with a horizontal solid line for the total lactation (DIM 8-330). Black lines indicate Swedish Holstein and gray lines indicate Swedish Red.

from the whole lactation (DIM 8-330). The heritability estimates for MT then increased as expected for both breeds, as did the heritability for AFR in SR, when data of the first and last parts of the lactation were excluded. The estimates for AFR and BT in SH did, however, stay at the same level, whereas the estimate for BT in SR decreased.
Correlations of cow EBV from the repeatability Model [3] with EBV from the RR3 model were close to unity, especially for AFR (0.95-0.99) and MT (0.950.99). Corresponding ranges for BT were 0.92 to 0.97 . The high correlations suggest that we would select the same cows whether the repeatability model or the RR model is used for genetic evaluation. Dodenhoff and Emmerling (2009) concluded that because the genetic correlations between test-day AFR were lower than unity, using a random regression test-day model, which describes the correlation structure more accurately, may improve the accuracy of selection. However, their results with high correlations between first-lactation test-days indicate that the possible advantage of using a random regression model may be smaller for milkability than for other dairy traits (Dodenhoff and Emmerling, 2009). Laureano et al. (2012) studied milkability based on monthly test-day flow rate records and compared the results from a multiple-trait model considering each test-day as a distinct trait with a repeatability model. The genetic correlations between monthly milk flow rates obtained by the multiple-trait analysis were high, suggesting that a repeatability model is able to fit the milkability data. The results from the present study are in agreement with those studies, with formal model tests in favor of a RR model, but the Pearson correlations indicate that the repeatability model used would give almost the same results for practical genetic evaluation purposes.

\section{CONCLUSIONS}

The present study showed high genetic correlations between milking systems (AMS and CMP) and indicated the potential joint use of data from AMS and CMP for genetic evaluation of milkability traits. High genetic correlations between lactations and high repeatabilities within and across lactations indicate that it should be sufficient to include milkability information from a few recordings in first lactation for the genetic evaluation and still improve milkability in later lactations. Because the different milkability traits were highly genetically correlated, it is necessary to include only one of the traits, either AFR or MT (BT), in the genetic evaluation. Model comparisons imply that genetic merit would be predicted most precisely with a random regression model but, in practice, only small differences exist between the use of a random regression model and a repeatability model.

\section{ACKNOWLEDGMENTS}

The authors acknowledge the Swedish Farmers' Foundation for Agricultural Research (Stockholm, 
Sweden) for their financial support. DeLaval (Tumba, Sweden) generously enabled contact with dairy herds using AMS. The included dairy herds and Växa Sweden (Stockholm, Sweden; previously named Swedish Dairy Association) are gratefully acknowledged for providing data for the study.

\section{REFERENCES}

Byskov, K., L. H. Buch, and G. P. Aamand. 2012. Possibilities of implementing measures from automatic milking systems in routine evaluations of udder conformation and milking speed. Interbull Bull. 46:28-32.

Carlström, C., G. Pettersson, K. Johansson, E. Strandberg, H. Stålhammar, and J. Philipsson. 2013. Feasibility of using milkability data from commercial automatic milking systems for genetic analysis. J. Dairy Sci. 96:5324-5332.

Dodenhoff, J., and R. Emmerling. 2009. Genetic parameters for milkability from the first three lactations in Fleckvieh cows. Animal 3:329-335.

Gäde, S., E. Stamer, J. Bennewitz, W. Junge, and E. Kalm. 2007. Genetic parameters for serial, automatically recorded milkability and its relationship to udder health in dairy cattle. Animal 1:787-796.

Gäde, S., E. Stamer, W. Junge, and E. Kalm. 2006. Estimates of genetic parameters for milkability from automatic milking. Livest. Sci. 104:135-146.
Gray, K. A., F. Vacirca, A. Bagnato, A. B. Samoré, A. Rossoni, and C. Maltecca. 2011. Genetic evaluations for measures of the milkflow curve in the Italian Brown Swiss population. J. Dairy Sci. 94:960-970.

Jensen, J., E. A. Mäntysaari, P. Madsen, and R. Thompson. 1997. Residual maximum likelihood estimation of (co)variance components in multivariate mixed linear models using average information. J. Ind. Soc. Agric. Stat. 49:215-236.

Laureano, M. M. M., A. B. Bignardi, L. El Faro, V. L. Cardoso, and L. G. Albuquerque. 2012. Genetic parameters for first lactation test-day milk flow in Holstein cows. Animal 6:31-35.

Løvendahl, P., J. Lassen, and M. G. G. Chagunda. 2011. Genetic analysis of milkability, milking frequency, milk yield and composition in automatically milked cows. Page 80 in Book of Abstracts of 62nd Annu. Meet. Eur. Fed. Anim. Sci., Stavanger, Norway. Wageningen Academic Publishers, Wageningen, the Netherlands.

Madsen, P., and J. Jensen. 2007. A User's Guide to DMU, A Package for Analyzing Multivariate Mixed Models. Version 6, release 4.7. University of Aarhus, Faculty Agricultural Sciences, Dept. of Genetics and Biotechnology, Research Centre Foulum, Denmark.

Norgren, A. 2005. Studier av mjölkbarhet automatiskt mätt i mjölkningsanläggningar (Study of milkability automatically measured in milking parlors). MS Thesis. Swedish University of Agricultural Sciences, Department of Animal Breeding and Genetics, Uppsala, Sweden.

Rensing, S., and W. Ruten. 2005. Genetic evaluation for milking speed in German Holstein population using different traits in a multiple trait repeatability model. Interbull Bull. 33:163-166. 